Analytically solvable Hamiltonians for quantum two-level systems and their dynamics

This content has been downloaded from IOPscience. Please scroll down to see the full text.

2014 J. Phys. A: Math. Theor. 47445302

(http://iopscience.iop.org/1751-8121/47/44/445302)

View the table of contents for this issue, or go to the journal homepage for more

Download details:

IP Address: 133.9.4.187

This content was downloaded on 21/10/2014 at 23:57

Please note that terms and conditions apply. 


\title{
Analytically solvable Hamiltonians for quantum two-level systems and their dynamics
}

\author{
A Messina ${ }^{1}$ and H Nakazato ${ }^{2}$ \\ ${ }^{1}$ Dipartimento di Fisica e Chimica dell'Università di Palermo, Via Archirafi, 36, \\ I-90123 Palermo, Italy \\ ${ }^{2}$ Department of Physics, Waseda University, Tokyo 169-8555, Japan \\ E-mail: hiromici@waseda.jp
}

Received 24 July 2014, revised 4 September 2014

Accepted for publication 5 September 2014

Published 20 October 2014

\begin{abstract}
A simple systematic way of obtaining analytically solvable Hamiltonians for quantum two-level systems is presented. In this method, a time-dependent Hamiltonian and the resulting unitary evolution operator are connected through an arbitrary function of time, furnishing us with new analytically solvable cases. The method is surprisingly simple, direct, and transparent and is applicable to a wide class of two-level Hamiltonians with no involved constraint on the input function. A few examples illustrate how the method leads to simple solvable Hamiltonians and dynamics.
\end{abstract}

Keywords: solvable model, quantum two-level system, time-dependent Hamiltonian

PACS numbers: 03.65.Aa, 03.67.Ac, 07.05.Dz

\section{Introduction}

Investigating the quantum dynamics of a spin-1/2 particle subjected to an arbitrary timedependent classical magnetic field is the updated version of old seminal problems. One example is that of a periodically driven two-level system exactly treated by Rabi [1] in the theory of nuclear magnetic resonance. Another example concerns the physical behavior of a quantum system around an avoided crossing region, analyzed with no approximation by Landau [2] and Zener [3] for a two-level system. The evergreen attractiveness of the problem under scrutiny is traceable back to the underlying Hamiltonian model, whose language is universal enough to describe scenarios of basic and applicative importance in many different physical contexts such as, in addition to those previously mentioned, superconductivity [4], quantum optics [5], quantum control [6], quantum information, and quantum computing [7]. 
It is, moreover, worth noting that mathematical results advancing our understanding of the solution of the general problem are useful to highlight aspects of the dynamics of a classical gyroscope under the action of the time-dependent magnetic field [8]. They are also useful to suggest new tricky ideas to provide a protocol to construct a class of exact solutions of a linear and homogeneous system of two coupled differential equations with time-dependent coefficients.

Unfortunately, only a few examples of analytically solvable two-level evolutions have been reported up to now, generally confined to very specific classes of time-dependent field configurations: mainly periodic [9] or pulse shaped [10]. Generally speaking, however, the physical behavior of a two-level system against the wide variety of the time-dependent magnetic fields acting upon it is very rich, exhibiting, for instance, intriguing resonance phenomena like the dynamical stabilization [11] (i.e., the tendency to occupy a fixed state during a finite period of time) or the coherent destruction of tunneling [12] (i.e., a remarkable diminution of the initial tunneling frequency).

It is thus remarkable that Barnes and Das Sarma [13] pointed out that if the problem is approached from a different direction, one could reach an unlimited number of solvable cases which could not otherwise be reached by the ordinary strategy. They considered a class of two-level Hamiltonians that are characterized by a time-dependent control field along the $z$ direction and a constant one along the $x$ direction. They succeeded in connecting the control field in the Hamiltonian and the ensuing evolution operator in terms of a free input function. Such a parametric connection is implicit; however, it really presents us with new solvable models.

In this paper, a systematic way of generating solvable Hamiltonians of quantum twolevel systems is presented. Though the essential idea is the same as in [13], i.e., to connect analytically the Hamiltonian and the resulting evolution operator in terms of an arbitrary parametric input function, the method here is much simpler and transparent, and the connection is direct, thus allowing us to have explicit expressions for both the Hamiltonian and the evolution operator. Furthermore, our approach enlarges the range of applicability of [13], allowing us to consider the most general form of Hamiltonian [14]. At the same time, since our approach automatically guarantees unitarity, the input function is free from any involved constraint that was necessary in $[13,14]$. It should be stressed that different parametrizations are possible, and the ones used in this paper give rise to solvable time-dependent problems not contained in the set stemming from the parametrization of $[13,14]$. Thus the treatment and solutions here presented will turn out to be a useful further resource complementary to those previously obtained.

\section{New strategy to generate solvable Hamiltonians}

Consider a quantum two-level system described by a general time-dependent Hamiltonian

$$
H=\left(\begin{array}{cc}
\Omega & \omega \\
\omega^{*} & -\Omega
\end{array}\right),
$$

where $\Omega(\omega)$ is a real (generally complex) function of $t$. This is the most general form of the hermitian two-by-two operator (besides an additive time-dependent term proportional to the unit operator 1 , which would trivially result in a time-dependent overall phase factor in the evolution operator). The unitary evolution operator generated by this Hamiltonian is an element of SU(2), which is always representable in terms of two complex functions, $a$ and $b$, as 


$$
U=\left(\begin{array}{ll}
a & b \\
-b^{*} & a^{*}
\end{array}\right), \quad|a|^{2}+|b|^{2}=1
$$

Since the evolution operator has to satisfy $\mathrm{i} \hbar \dot{U}=H U$ or, equivalently, $H=\mathrm{i} \hbar \dot{U} U^{\dagger}$, the functions in equations (1) and (2) are related to each other as follows

$$
\mathrm{i} \hbar\left(\dot{a} a^{*}+\dot{b} b^{*}\right)=\Omega, \quad \mathrm{i} \hbar(a \dot{b}-\dot{a} b)=\omega .
$$

Observe first that the second equation is solved to express $b$ in terms of $a$ and $\omega$

$$
b=\frac{a}{\mathrm{i} \hbar} \int_{0}^{t} \frac{\omega}{a^{2}},
$$

where the initial condition

$$
U(0)=1 \quad \text { or } \quad a(0)=1, \quad b(0)=0
$$

has been incorporated. (Here and in the following, integration variables have been and will be completely suppressed if no confusion would arise.) Elimination of $b$ thus results in a closed form of (integro-differential) equation for $a$

$$
\dot{a}=-\frac{\omega}{\hbar^{2}} X^{*} a^{*}+\frac{\Omega}{\mathrm{i} \hbar} a, \quad X \equiv \int_{0}^{t} \frac{\omega}{a^{2}},
$$

where the function $X$ is constrained to satisfy

$$
|a|^{2}\left[1+\frac{1}{\hbar^{2}}|X|^{2}\right]=1 \text {. }
$$

Usually one tries to find the evolution operator $U$ (equivalently, the functions $a$ and $b$ ) in terms of the entries $\Omega$ and $\omega$ in (1). Unfortunately, it is in general difficult to find exactly solvable cases other than the well-known ones. If, however, we limit this general target searching for any longitudinal $\Omega(t)$ (transverse $\omega(t)$ ), possible forms of $\omega(t)(\Omega(t)$ ) by which the dynamical problem is exactly solvable, we disclose the existence of physical scenarios wherein the dynamical behavior of the two-level systems may be fully investigated with no approximation.

It is proposed here to view the above equation (6) and the constraint (7) as those prescribing $a$ and $\omega$ once the functions $X$ and $\Omega$ have been given. Notice that the complex function $X(t)$ is completely arbitrary, provided it satisfies the initial condition $X(0)=0$. Since $\omega=a^{2} \dot{X}$ and $|a|^{2}=\left[1+|X|^{2} / \hbar^{2}\right]^{-1}$, the integro-differential equation (6) for $a$ becomes a differential equation

$$
\dot{a}=-\left(\frac{\mathrm{i}}{\hbar} \Omega+\frac{\dot{X} X^{*}}{\hbar^{2}+|X|^{2}}\right) a,
$$

which is solved to yield

$$
a=\frac{\hbar}{\left(\hbar^{2}+|X|^{2}\right)^{1 / 2}} \mathrm{e}^{-\frac{\mathrm{i}}{\hbar} \int_{0}^{t} \Omega-\mathrm{i} \int_{0}^{t} \frac{\Im\left(\dot{X} X^{*}\right)}{\hbar^{2}+|X|^{2}}}
$$

Once $a$ has been determined, the remaining functions are obtained from the relations

$$
b=\frac{1}{\mathrm{i} \hbar} a X, \quad \omega=a^{2} \dot{X} .
$$

The result means that if the transverse field $\omega(t)$ is given by this relation for an arbitrarily chosen $X(t)$ and an arbitrary longitudinal field $\Omega(t)$, the resulting unitary evolution operator is 
exactly given by the parameter $a(t)$ in (9) and $b(t)$ in (10). The relations are exact, and no approximation is involved. We emphasize that the introduction of the function $X(t)$ as given by (6) is indeed the mathematical trick originating $t$-dependent Hamiltonian models for which simultaneous explicit calculation of the evolution operator is straightforward. Such an auxiliary function $X(t)$ allows us indeed to link implicitly the two entries of $H$ with those of $U$, right in view of (9) and (10) expressing $a, b$, and $\omega$ in terms of $X$ and $\Omega$.

It would be worth stressing that an approach different from the ordinary strategy can result in establishing an exact relationship between the Hamiltonian and the dynamics, even though the derivation itself is straightforward and simple. In this approach, a single complex function $X$ plays the key role, and no other function is required, while in [13] several functions were introduced to establish the connection. Furthermore, the unitarity of the evolution operator, expressed as (7), has been taken into account when the parameter $a$ is solved in (9), and thus no further constraints are necessary. Recall again that we consider the most general form of $H$, i.e., (1) (apart from a term proportional to the identity operator, which would result in an additional overall phase factor), so that the method is applicable to any two-level system scenario.

\section{Selective examples}

A few examples would be helpful to understand how the method works as well as its usefulness in practice.

\subsection{Example 1: a real function $X$}

When one chooses a real function $X, X=X^{*}$ and specifies the transverse field as

$$
\omega=\frac{\hbar^{2} \dot{X}}{\hbar^{2}+|X|^{2}} \mathrm{e}^{-\frac{2 \mathrm{i}}{\hbar} \int_{0}^{t} \Omega},
$$

the evolution operator is characterized by the parameters

$$
a=\frac{\hbar \mathrm{e}^{-\frac{\mathrm{i}}{\hbar} \int_{0}^{t} \Omega}}{\left(\hbar^{2}+|X|^{2}\right)^{1 / 2}}, \quad b=\frac{-\mathrm{i} X \mathrm{e}^{-\frac{\mathrm{i}}{\hbar} \int_{0}^{t} \Omega}}{\left(\hbar^{2}+|X|^{2}\right)^{1 / 2}} .
$$

Notice that the above equation (11) can be integrated to give $X$ in terms of $\omega$ and $\Omega$

$$
X=\hbar \tan \left[\int_{0}^{t} \frac{\omega}{\hbar} \mathrm{e}^{\frac{2 \mathrm{i}}{\hbar} \int_{0}^{t^{\prime}} \Omega}\right]
$$

The consistency requires that the integrand in the brackets should be a real function, which implies that the interaction Hamiltonian in the interaction picture commutes at different times; that is, this case corresponds to a well-known solvable case [15].

\subsection{Example 2: a complex function $X$}

Consider a complex function $X(t)$ parametrized by a real function $\phi(t)$ and a real constant parameter $c$ as

$$
X=c \sin \phi \mathrm{e}^{\mathrm{i} \phi}, \quad \phi(0)=0 .
$$


The general formula tells us that when the transverse field is prescribed as

$$
\omega=\frac{\hbar^{2} c \dot{\phi}}{\hbar^{2}+c^{2} \sin ^{2} \phi} \mathrm{e}^{-\frac{2 \mathrm{i}}{\hbar} \int_{0}^{t} \Omega+2 \mathrm{i} \int_{0}^{t} \frac{\hbar^{2} \dot{\phi}}{\hbar^{2}+c^{2} \sin ^{2} \phi},}
$$

we are led to an exact solution

$$
a=\frac{\hbar}{\sqrt{\hbar^{2}+c^{2} \sin ^{2} \phi}} \mathrm{e}^{-\frac{\mathrm{i}}{\hbar} \int_{0}^{t} \Omega-\mathrm{i} \int_{0}^{t} \frac{c^{2} \dot{\phi} \sin ^{2} \phi}{\hbar^{2}+c^{2} \sin ^{2} \phi}}, \quad b=\frac{1}{\mathrm{i} \hbar} a X .
$$

Observe that equation (15) requires that ( $c>0$ and $\dot{\phi}>0$ are assumed for simplicity) $|\omega|=\frac{c \hbar^{2} \dot{\phi}}{\hbar^{2}+c^{2} \sin ^{2} \phi}, \phi_{\omega}=-\frac{2}{\hbar} \int_{0}^{t} \Omega+2 \int_{0}^{t} \frac{\hbar^{2} \dot{\phi}}{\hbar^{2}+c^{2} \sin ^{2} \phi}$. In other words, if the magnitude and the phase of the transverse field $\omega \equiv|\omega| \mathrm{e}^{\mathrm{i} \phi_{\omega}}$ are inter-connected as

$$
\frac{|\omega(t)|}{c}=\frac{\Omega(t)}{\hbar}+\frac{\dot{\phi}_{\omega}(t)}{2},
$$

we are led to exact solutions. This certainly enlarges the domain of solvability, since if we take the $c=\infty$ limit while keeping finite $X \neq 0$ and $|\omega| \neq 0$ to nullify the right-hand side of (17), we obtain the case where the interaction Hamiltonian in the interaction picture is commutable at different times, leading to a known exact solution [15]. Now we understand that there are more solvable cases. Indeed it is not difficult to express $\phi$ in terms of $|\omega|$

$$
\tan \phi=\frac{\hbar}{\sqrt{\hbar^{2}+c^{2}}} \tan \Phi(t), \quad \Phi(t)=\frac{\sqrt{\hbar^{2}+c^{2}}}{\hbar c} \int_{0}^{t}|\omega| .
$$

This means that, given arbitrary functions $\Omega$ and $|\omega|$, we control the phase $\phi_{\omega}$ as

$$
\phi_{\omega}=\frac{2}{c} \int_{0}^{t}|\omega|-\frac{2}{\hbar} \int_{0}^{t} \Omega
$$

then the unitary evolution is explicitly given by

$$
\begin{aligned}
& a=\sqrt{\frac{\hbar^{2}+c^{2} \cos ^{2} \Phi}{\hbar^{2}+c^{2}}} \mathrm{e}^{-\frac{\mathrm{i}}{\hbar} \int_{0}^{t} \Omega+\frac{\mathrm{i}}{c} \int_{0}^{t}|\omega|-\mathrm{i} \tan ^{-1}\left(\frac{\hbar}{\sqrt{\hbar^{2}+c^{2}}} \tan \Phi\right),} \\
& b=-\mathrm{i} \frac{c \sin \Phi}{\sqrt{\hbar^{2}+c^{2}}} \mathrm{e}^{-\frac{\mathrm{i}}{\hbar} \int_{0}^{t} \Omega+\frac{\mathrm{i}}{c} \int_{0}^{t}|\omega|} .
\end{aligned}
$$

We stress here that this simple case is to be added as a new category of solvable Hamiltonians to the well-known cases where the interaction Hamiltonian in the interaction picture is commutable at different times. Notice that in this case the input function $X(t)$ can be eliminated, and the relationship between the parameters in the Hamiltonian, $\Omega(t)$ and $\omega(t)$, and those characterizing the evolution operator $U(t), a(t)$, and $b(t)$, has become explicit. That is, this case really presents us with analytically solvable Hamiltonians in the ordinary sense.

\subsection{An arbitrary complex function $X$}

In the above examples, one of the two degrees of freedom of $X$ has been suppressed or eliminated, leading to simple expressions of parameters $\omega, a$, and $b$. To treat the most general form of $X$, we keep both degrees of freedom and introduce polar variables $X=A \mathrm{e}^{\mathrm{i} \phi}$ with two real functions $A$ and $\phi$, assuming $A(0)=0$. The relation $\omega=a^{2} \dot{X}$ implies that the angle variable $\phi$ has to satisfy 


$$
\dot{\phi}=\frac{\dot{A}}{A} \tan \left\{\phi_{\omega}-\phi+2 \int_{0}^{t}\left(\frac{\Omega}{\hbar}+\frac{\dot{\phi} A^{2}}{\hbar^{2}+A^{2}}\right)\right\} \equiv \frac{\dot{A}}{A} \tan \Theta
$$

and therefore

$$
\dot{A}^{2}+\dot{\phi}^{2} A^{2}=\dot{A}^{2}\left(1+\tan ^{2} \Theta\right)=|\omega|^{2}\left(1+A^{2} / \hbar^{2}\right)^{2} .
$$

This differential equation is solved for $A$

$$
A=\hbar \tan \left[\frac{1}{\hbar} \int_{0}^{t}|\omega| \cos \Theta\right]
$$

and we obtain

$$
\dot{\phi}=\frac{\dot{A}}{A} \tan \Theta=\frac{2|\omega|}{\hbar} \frac{\sin \Theta}{\sin \left[\frac{2}{\hbar} \int_{0}^{t}|\omega| \cos \Theta\right]} .
$$

Observe that we have to put $\sin \Theta(0)=0$ if we want to keep parameters well behaved. These variables are constrained to satisfy $\Theta=\phi_{\omega}-\phi+2 \int_{0}^{t}\left(\frac{\Omega}{\hbar}+\frac{\dot{\phi} A^{2}}{\hbar^{2}+A^{2}}\right)$ or

$$
\begin{aligned}
\dot{\Theta} & =\frac{2 \Omega}{\hbar}+\frac{A^{2}-\hbar^{2}}{A^{2}+\hbar^{2}} \dot{\phi}+\dot{\phi}_{\omega} \\
& =\frac{2 \Omega}{\hbar}-\frac{2|\omega|}{\hbar} \sin \Theta \cot \left[\frac{2}{\hbar} \int_{0}^{t}|\omega| \cos \Theta\right]+\dot{\phi}_{\omega} .
\end{aligned}
$$

This equation suggests to exchange, in our general framework, the roles of $\Omega$ and $\omega$. It is indeed advantageous to view the relation (25) as that prescribing $\Omega$ in terms of $\omega=|\omega| \mathrm{e}^{\mathrm{i} \phi_{\omega}}$ and $\Theta$,

$$
\Omega=\frac{\hbar}{2}\left(\dot{\Theta}-\dot{\phi}_{\omega}\right)+|\omega| \sin \Theta \cot \left[\frac{2}{\hbar} \int_{0}^{t}|\omega| \cos \Theta\right]
$$

and in this case we obtain an exact expression of $a$

$$
a=\cos \left[\frac{1}{\hbar} \int_{0}^{t}|\omega| \cos \Theta\right] \exp \left\{-\mathrm{i} \int_{0}^{t}\left(\frac{\dot{\Theta}-\dot{\phi}_{\omega}}{2}+\frac{|\omega| \sin \Theta}{\hbar \sin \left[\frac{2}{\hbar} \int_{0}^{t^{\prime}}|\omega| \cos \Theta\right]}\right]\right\}
$$

That is, for any transverse field $\omega=|\omega| \mathrm{e}^{\mathrm{i} \phi_{\omega}}$, if the longitudinal field $\Omega$ is controlled as in (26), the resulting dynamics is exactly given by this parameter $a$, and $b=a A \mathrm{e}^{\mathrm{i} \phi} /(\mathrm{i} \hbar)$, where the real function $\Theta$ can be chosen as we wish and $A$ and $\phi$ have already been given as functions of $\omega$ and $\Theta$ (see (23) and (24)).

These exact expressions could present us with interesting solutions that otherwise could never be found. On the mathematical side we claim that any successful application based on the protocol here reported also provides direct tools to find straightforwardly exact, closed, and explicit solutions of the otherwise generally intractable homogeneous system of the two linear differential equations, corresponding to the time-dependent Schrödinger equation associated to $H$ in (1). 


\subsection{Example 3: constant transverse field $\omega=$ const.}

In what follows, we consider the cases in which the transverse field is constant, $|\omega|=$ const.,$\dot{\phi}_{\omega}=0$. These are the cases just considered in [13]; however, all the expressions here, which have never been obtained before, are rather simple and instructive, and the evolution operator can be expressed in terms of elementary analytical functions.

3.4.1. Monotonically decreasing $|\mathbf{a}|$. We may put

$$
\frac{|\omega|}{\hbar} \int_{0}^{t} \cos \Theta=\frac{\pi}{4} \tanh \gamma t, \quad \gamma=\frac{4|\omega|}{\pi \hbar} .
$$

In this case, we can choose

$$
\cos \Theta=\cosh ^{-2} \gamma t, \quad \sin \Theta=\frac{\tanh \gamma t}{\cosh \gamma t} \sqrt{\cosh ^{2} \gamma t+1}, \quad \dot{\Theta}=\frac{2 \gamma}{\cosh \gamma t \sqrt{\cosh ^{2} \gamma t+1}},
$$

and therefore the longitudinal field, engineered as

$$
\Omega=\frac{\gamma \hbar}{\cosh \gamma t \sqrt{\cosh ^{2} \gamma t+1}}+|\omega| \frac{\tanh \gamma t \sqrt{\cosh ^{2} \gamma t+1}}{\cosh \gamma t} \cot \left[\frac{\pi}{2} \tanh \gamma t\right]
$$

entails a unitary evolution that is characterized by its diagonal and off-diagonal matrix elements

$$
|a|=\cos \left[\frac{\pi}{4} \tanh \gamma t\right], \quad|b|=\sin \left[\frac{\pi}{4} \tanh \gamma t\right],
$$

and, for $t \gg \gamma^{-1}=\frac{\pi \hbar}{4|\omega|}$, approaches its asymptotic behavior

$$
\begin{aligned}
U & =\frac{1}{\sqrt{2}}\left(\begin{array}{ll}
\mathrm{e}^{\mathrm{i} \phi_{a}} & \mathrm{e}^{\mathrm{i} \phi_{b}} \\
-\mathrm{e}^{-\mathrm{i} \phi_{b}} & \mathrm{e}^{-\mathrm{i} \phi_{a}}
\end{array}\right), \\
\phi_{b}-\phi_{a} & =\frac{2|\omega|}{\hbar} \int_{0}^{t} \frac{\tanh \gamma t^{\prime} \sqrt{\cosh ^{2} \gamma t^{\prime}+1}}{\cosh \gamma t^{\prime} \sin \left[\frac{\pi}{2} \tanh \gamma t^{\prime}\right]}-\frac{\pi}{2} .
\end{aligned}
$$

Notice that the engineered longitudinal field $\Omega$, together with the constant transverse field $\omega$, ensures this asymptotic form of the unitary evolution for all t after the transient time $\gamma^{-1}$, while a constant transverse field $\omega$ with a vanishing $\Omega$, for example, brings about an oscillating behavior between the diagonal and off-diagonal elements. The unitary evolution (32) may be easily generalized by simply substituting $\frac{\pi}{4}$ in (28) with a generic angle, realizing in this way a superposition of $\left(\begin{array}{l}1 \\ 0\end{array}\right)$ and $\left(\begin{array}{l}0 \\ 1\end{array}\right)$ with a specific relative weight without adjusting the evolution time: one just needs to wait for a long time.

3.4.2. Constant $|\boldsymbol{a}|$ for a finite time interval. Consider abrupt switchings of the parameter $\Theta$, an ideal form of which may be given by

$$
\Theta=\frac{\pi}{4}\left[\epsilon\left(t-T_{1}\right)+\epsilon\left(T_{2}-t\right)\right], \quad\left(0<T_{1}<T_{2}\right),
$$

where $\epsilon$ is a sign function $\epsilon(x)=\theta(x)-\theta(-x), \theta(x)$ being the Heaviside function. We can halt the oscillating behavior of $|a|$ at $t=T_{1}$ for a finite duration $T_{1}<t<T_{2}$ 


$$
a= \begin{cases}\cos \frac{|\omega| t}{\hbar}, & \left(0 \leqslant t<T_{1}\right), \\ \mathrm{e}^{-\frac{\pi i}{4}-\frac{\mathrm{i}|\omega|\left(t-T_{1}\right)}{\hbar \sin \left(2|\omega| T_{1} / \hbar\right)} \cos \frac{|\omega| T_{1}}{\hbar},} & \left(T_{1}<t<T_{2}\right), \\ \mathrm{e}^{-\frac{\mathrm{i}|\omega|\left(T_{2}-T_{1}\right)}{\hbar \sin \left(2|\omega| T_{1} / \hbar\right)} \cos \frac{|\omega|\left(t-T_{2}+T_{1}\right)}{\hbar},} & \left(T_{2}<t\right) .\end{cases}
$$

In order to realize this behavior, we have to control the longitudinal field as

$$
\Omega=\frac{\pi \hbar}{4}\left[\delta\left(t-T_{1}\right)-\delta\left(t-T_{2}\right)\right]+\theta\left(T_{2}-t\right) \theta\left(t-T_{1}\right)|\omega| \cot \frac{2|\omega| T_{1}}{\hbar} .
$$

It is interesting to see exactly that the otherwise oscillating behavior of the unitary evolution operator can be stopped by the application of an impulsive longitudinal field; then the magnitude of its matrix elements can be kept constant for a finite time duration. This mechanism would bring us a new possibility of controlling the dynamics at our disposal.

Of course, the appearance of the delta functions in $\Omega$ is due to our choice of ideal, i.e., instantaneous switching for $\Theta$. We may introduce a finite switching time and, accordingly, the relevant quantities would have to be modified. We underline that the essential feature, that is, the fact that the oscillating behavior of the unitary evolution stemming from the constant transverse field can be halted by the application of an engineered impulsive longitudinal one, would not be changed even after this kind of realistic modification.

3.4.3. A perfect inversion of the probabilities. It is possible to achieve a perfect inversion of the probabilities on the basis of our exact solution. One may choose $T_{1}=\frac{\pi \hbar}{2|\omega|}$ and $T_{2}=\infty$ in the above example to realize a completely off-diagonal form of $U$ at $t \geqslant T_{1}$. The longitudinal field $\Omega$ has to be controlled as in (35) with $T_{2}=\infty$. Another choice would be

$$
\cos \Theta=\cosh ^{-2} \tilde{\gamma} t, \quad \tilde{\gamma}=\frac{2|\omega|}{\pi \hbar} .
$$

Observing the similarity to the previous example 3.4.1, we understand that the control longitudinal field $\Omega$ is given by the similar expression as in (30), with the factors $\gamma$ and $\frac{\pi}{2}$ now replaced by $\tilde{\gamma}$ and $\pi$, respectively, and

$$
|a|=\cos \left[\frac{\pi}{2} \tanh \tilde{\gamma} t\right], \quad|b|=\sin \left[\frac{\pi}{2} \tanh \tilde{\gamma} t\right] .
$$

Notice that the control longitudinal field $\Omega$ behaves asymptotically as $\Omega(\infty)=$ $|\omega| \cot \pi=-\infty$, while $\Omega(0)=\frac{2 \sqrt{2}}{\pi}|\omega|$. In either case, since the unitary operator $U$ becomes completely off diagonal at $t=T_{1}$ or $t=\infty(a=0)$, the probabilities of being up or down states at $t=0$ are perfectly interchanged at $t=T_{1}$ or $t=\infty$.

These transition processes remind us of the famous Landau-Zener transition [2, 3], where the longitudinal field $\Omega$ is assumed to vary linearly in $t$, and the non-adiabatic transition between $t=-\infty$ and $t=\infty$ is unavoidable as long as $\Omega$ changes with a nonvanishing speed. In the present framework, it is shown explicitly that a choice $\cos \Theta=\alpha \mathrm{e}^{-\gamma^{\prime}|t|} \leqslant \alpha \equiv \frac{\pi \hbar}{4|\omega|} \gamma^{\prime}<1$, for example, certainly results in a perfect transition of the lowest eigenstate of the Hamiltonian at $t=-\infty,\left(\begin{array}{l}0 \\ 1\end{array}\right)$, to that of $H(\infty),\left(\begin{array}{l}1 \\ 0\end{array}\right)$, at $t=\infty$, with probability one [14]. 


\section{Discussions and conclusions}

The examples presented above clearly show how simply and easily the present method can provide us with novel analytically solvable Hamiltonians and their dynamics that can be expressed directly and explicitly in terms of elementary functions. Notice that to obtain solvable Hamiltonians (in a broader sense) for two-level systems practically means to establish an analytical relationship between three input parameters and three output parameters. So far we have tried to express the three parameters in the unitary evolution operator $a(t)$ and $b(t)$ with the constraint $|a(t)|^{2}+|b(t)|^{2}=1$ in terms of those in the Hamiltonian $\Omega(t)=\Omega^{*}(t)$ and $\omega(t)=|\omega(t)| \mathrm{e}^{\mathrm{i} \phi_{\omega}(t)}$. It is well known that this strategy works when $\dot{\phi}_{\omega}=-2 \Omega / \hbar$, which constitutes the solvable case in the ordinary sense.

Now we know that there are other strategies (in the broader sense), and we may choose different combinations of three parameters as inputs. Here we consider essentially the following two alternative strategies: (1) given the transverse field $\omega$ and one parameter $\Theta$, to find the unitary operator under the engineered longitudinal field $\Omega$, and (2) given the longitudinal field $\Omega$ and a complex parameter $X$, to find the evolution operator under the engineered transverse field $\omega$. The previous studies $[13,14]$ are along the line of strategy (1), while the present method applies to both (1) and (2). Actually, we have started with (2) and moved to (1) in this paper.

Consider strategy (1) first. The input parameters in [14] are denoted as $\beta, \varphi$, and $\chi$, while the complex $\omega$ and real $\Theta$ are the three input parameters in the present method. It is not difficult to find the following correspondence between them, i.e., $\beta=|\omega|, \varphi=-\phi_{\omega}$, $\dot{\chi}=-|\omega| \cos \Theta(\hbar=1)$. It is clear that $\dot{\chi}$ has to satisfy the constraint $1-\dot{\chi}^{2} / \beta^{2} \geqslant 0$, while the parameter $\Theta$ is free from it. We understand that the present parametrization is much more suitable and tractable, since to find an appropriate $\chi$ seems quite nontrivial and difficult.

Strategy (2) is not considered in $[13,14]$ and is the basis of the present method, where the longitudinal field $\Omega$ and a complex function $X$ are the input parameters that one can take at one's will. In this respect, the new solvable case found in example 2 is unique and deserved to be stressed. Actually, this case has revealed the fact for the first time that the dynamics can be solved analytically in the ordinary sense, even if the commutativity of the interaction Hamiltonian in the interaction picture at different times is not met, provided the parameters in the Hamiltonian satisfy the relation (19).

The present method would open a new door to find analytically solvable Hamiltonians in two-level quantum systems and to control such systems where the dynamics can be predicted analytically. It is, of course, possible to consider a different input function $X$ or $\Theta$ to apply the method in different contexts, which will be presented elsewhere.

\section{Acknowledgments}

This work is partially supported by a Grant-in-Aid for Scientific Research (C) (No. 22540292) from JSPS.

\section{References}

[1] Rabi I I 1937 Phys. Rev. 51652

[2] Landau L 1932 Phys. Z. Sowjetunion 246

[3] Zener C 1932 Proc. R. Soc. A 137696 
[4] Gangopadhyay A 2012 Theoretical studies of the interplay between superconductivity and disorder PhD Thesis University of Maryland

[5] Haroche S and Raimond J-M 2006 Exploring the Quantum: Atoms Cavities And Photons (Oxford: Oxford University Press)

[6] Daems D, Ruschhaupt A, Sugny D and Guerin S 2013 Phys. Rev. Lett. 111050404 (arXiv:1304.4016v2)

Greilich A, Economou S E, Spatzek S, Yakovlev D R, Reuter D, Wieck A D, Reinecke T L and Bayer M 2009 Nature Phys. 5262

Poem E, Kenneth O, Kodriano Y, Benny Y, Khatsevich S, Avron J E and Gershoni D 2011 Phys. Rev. Lett. 107087401

[7] Nielsen M A and Chuang I L 1990 Quantum Computation and Quantum Information (Cambridge: Cambridge University Press)

Ashhab S, Johansson J R, Zagoskin A M and Nori F 2007 Phys. Rev. A 75063414

Bouwmeester D, Ekert A and Zeilinger A 2000 The Physics of Quantum Information: Quantum Cryptography, Quantum Teleportation, Quantum Computation (Berlin: Springer)

Gerardot B D and Öhberg P 2009 Science 3261489

Fuchs G D, Dobrovitski V V, Toyli D M, Heremans F J and Awschalom D D 2009 Science 3261520

[8] Feynman R P, Vernon F L Jr. and Hellwarth R W 1957 J. Appl. Phys. 2849

[9] Bezvershenko Y V and Holod P I 2011 Phys. Lett. A 3753936

Gangopadhyay A, Dzero M and Galitski V 2010 Phys. Rev. B 82024303

Barata J C A and Cortez D A 2002 Phys. Lett. A 301350 (arXiv:0202110v2)

Xie Q and Hai W 2010 Phys. Rev. A 82032117

[10] Rosen N and Zener C 1932 Phys. Rev. 40502

Vitanov N V 2007 New J. Phys. 958 and references therein

[11] Bezvershenko Y V, Holod P I and Messina A 2011 Phys. Scr. T143 014005

[12] Grossmann F, Dittrich T, Jung P and Hänggi P 1991 Phys. Rev. Lett. 67516

[13] Barnes E and Das Sarma S 2012 Phys. Rev. Lett. 109060401

[14] Barnes E 2013 Phys. Rev. A 88013818

[15] Sakurai J J 1994 Modern Quantum Mechanics (Reading, MA: Addison-Wesley) 